\title{
Theory of tunneling spectroscopy of normal metal/ferromagnet/spin-triplet superconductor junctions
}

\author{
L. A. B. Olde Olthof, ${ }^{1}$ S.-I. Suzuki, ${ }^{2}$ A. A. Golubov, ${ }^{1,3}$ M. Kunieda, ${ }^{4}$ S. Yonezawa, ${ }^{4}$ Y. Maeno, ${ }^{4}$ and Y. Tanaka ${ }^{2}$ \\ ${ }^{1} \mathrm{MESA}^{+}$Institute for Nanotechnology, University of Twente, Enschede, Netherlands \\ ${ }^{2}$ Department of Applied Physics, Nagoya University, Nagoya 464-8603, Japan \\ ${ }^{3}$ Moscow Institute of Physics and Technology, Dolgoprudny, Moscow Region 141700, Russia \\ ${ }^{4}$ Department of Physics, Graduate School of Science, Kyoto University, Kyoto 606-8502, Japan
}

(Received 23 April 2018; revised manuscript received 25 June 2018; published 13 July 2018)

\begin{abstract}
We study the tunneling conductance of a ballistic normal metal/ferromagnet/spin-triplet superconductor junction using the extended Blonder-Tinkham-Klapwijk formalism as a model for a $c$-axis-oriented $\mathrm{Au} / \mathrm{SrRuO}_{3} / \mathrm{Sr}_{2} \mathrm{RuO}_{4}$ junction. We compare chiral $p$-wave (CPW) and helical $p$-wave (HPW) pair potentials, combined with ferromagnet magnetization directions parallel and perpendicular to the interface. For fixed $\theta_{M}$, where $\theta_{M}$ is a direction of magnetization in the ferromagnet measured from the $c$ axis, the tunneling conductances of CPW and HPW clearly show different voltage dependencies. It is found that the cases where the $d$ vector is perpendicular to the magnetization direction (CPW with $\theta_{M}=\pi / 2$ and HPW with $\theta_{M}=0$ ) are identical. The obtained results serve as a guide to determine the pairing symmetry of the spin-triplet superconductor $\mathrm{Sr}_{2} \mathrm{RuO}_{4}$.
\end{abstract}

DOI: 10.1103/PhysRevB.98.014508

\section{INTRODUCTION}

Nowadays, $\mathrm{Sr}_{2} \mathrm{RuO}_{4}$ is known as an unconventional superconductor with transition temperature $T_{c} \sim 1.5 \mathrm{~K}$ [1]. The fact that the Knight shift does not change across $T_{c}$ is consistent with spin-triplet pairing [2-6]. Various theoretical studies have discussed the microscopic mechanism of spin-triplet pairings in this material [7-21]. The existence of a zero-bias conductance peak in several tunneling experiments $[22,23]$ indicates the realization of unconventional superconductivity [24-26]. In particular, the broad zero-bias conductance peak observed in tunneling spectroscopy suggests the realization of a surface Andreev bound state (SABS) with linear dispersion [26-29]. This is in contrast to high- $T_{c}$ cuprate superconductors, in which a sharp zero-bias conductance peak is observed [24,25,30-35] due to flat-band zero-energy states [24,36,37]. When spintriplet pairing is realized, we can expect exotic phenomena, such as the so-called anomalous proximity effect in diffusive normal metal/spin-triplet superconductor junctions [38-42].

The presence or absence of time-reversal symmetry (TRS) in $\mathrm{Sr}_{2} \mathrm{RuO}_{4}$ is an important issue. Among two-dimensional spin-triplet $p$-wave pairings, chiral and helical $p$-wave pairings seem promising in the absence and presence of TRS, respectively [43]. Broken TRS was observed in muon spin-relaxation measurements ( $\mu \mathrm{SR})$ and Kerr-rotation experiments as a result of a spontaneous internal magnetic field below $T_{c}$ [44-46], which supports chiral $p$-wave pairing. However, the internal magnetic field has not been detected in scanning superconducting quantum interference device experiments $[47,48]$, which suggests realization of helical $p$-wave symmetry. Although there are several possible explanations for the absence of broken TRS in $\mathrm{Sr}_{2} \mathrm{RuO}_{4}$ [17,49-55], the pairing symmetry remains a point of discussion. One of the main differences between these two pairing symmetries is the direction of the $d$ vector.
A constructive way to distinguish between them is to study the charge transport in ferromagnet/spin-triplet superconductor junctions [56-60]. Naively speaking, the direction of the magnetization axis with respect to the $d$ vector (parallel or perpendicular) influences the charge transport. Recently, a $\mathrm{Au} / \mathrm{SrRuO}_{3} / \mathrm{Sr}_{2} \mathrm{RuO}_{4}$ junction oriented along the $c$ axis has been fabricated by means of epitaxial growth [61]. Since $\mathrm{SrRuO}_{3}$ and $\mathrm{Sr}_{2} \mathrm{RuO}_{4}$ have similar $a$-axis lattice constants, as well as similar atomic arrangements, a smooth interface between them can be expected, which turns this system into a nice playground for clarifying the direction of the $d$ vector. Because the SABS is absent in this direction, we can directly compare the effect of the magnetization direction relative to the $d$ vector. To interpret the experimental results, a theoretical model is required in which we calculate the tunneling conductance along the $c$ axis based on a minimal model which takes the quasi-two-dimensional nature of $\mathrm{Sr}_{2} \mathrm{RuO}_{4}$ into account.

In this paper, we investigate normal metal $(\mathrm{N}) /$ ferromagnet (F)/spin-triplet superconductor (S) junctions with $s$-wave and chiral and helical $p$-wave pairing symmetries by changing the properties of the ferromagnet, e.g., thickness, magnetization strength, and direction. The anisotropic Fermi surface of $\mathrm{Sr}_{2} \mathrm{RuO}_{4}$ and realistic effective masses are also included since the Fermi-momentum mismatch changes the transparency and the resulting conductance. Finally, an external magnetic field is taken into account through the Doppler shift.

\section{FORMULATION}

\section{A. Model and Hamiltonian}

We consider a three-dimensional N/F/S junction, as shown in Fig. 1. We assume the junction interfaces are perpendicular to the $z$ axis and located at $z=0$ and $z=L$. The $\mathrm{F}$ has a thickness $L$ and a magnetization $\boldsymbol{M}(z)$. The $\mathrm{N}$ and $\mathrm{S}$ are 


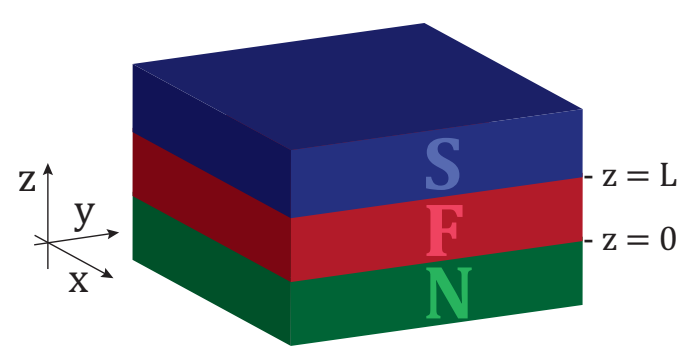

FIG. 1. Schematic of the three-dimensional normal metal (N)/ ferromagnet $(\mathrm{F}) /$ superconductor $(\mathrm{S})$ junction. We assume the structure extends to infinity in all directions.

considered to be semi-infinite. Superconducting junctions are described by the Bogoliubov-de Gennes (BdG) Hamiltonian

$$
\check{\mathcal{H}}(\boldsymbol{r})=\left[\begin{array}{cc}
\hat{h}(\boldsymbol{r}, H) & \hat{\Delta}(\boldsymbol{r}) \\
-\hat{\Delta}^{*}(\boldsymbol{r}) & -\hat{h}^{*}(\boldsymbol{r}, H)
\end{array}\right],
$$

where the basis is taken to be $\Psi(\boldsymbol{r})=$ $\left[\psi_{\uparrow}(\boldsymbol{r}) \psi_{\downarrow}(\boldsymbol{r}) \psi_{\uparrow}^{\dagger}(\boldsymbol{r}) \psi_{\downarrow}^{\dagger}(\boldsymbol{r})\right]^{T}$, where $T$ is the transpose, the symbol $\hat{\sim}(\cdot)$ represents a $2 \times 2(4 \times 4)$ matrix in the spin (spin-Nambu) space, and $H$ is an externally applied magnetic field in the $x$ direction. Since the system has translational symmetry in the $x$ and $y$ directions, the momenta $k_{x}$ and $k_{y}$ are well-defined quantum numbers. Therefore, the wave function can be expressed in the Fourier components as

$$
\begin{gathered}
\Psi(\boldsymbol{r})=\sum_{\boldsymbol{k}_{\|}} \Psi_{\boldsymbol{k}_{\|}}(z) \frac{e^{i\left(k_{x} x+k_{y} y\right)}}{\sqrt{L_{x} L_{y}}}, \\
\Psi_{\boldsymbol{k}_{\|}}(z)=\left[\psi_{\uparrow, \boldsymbol{k}_{\|}} \psi_{\downarrow, \boldsymbol{k}_{\|}} \psi_{\uparrow,-\boldsymbol{k}_{\|}}^{\dagger} \psi_{\downarrow,-\boldsymbol{k}_{\|}}^{\dagger}\right]^{T},
\end{gathered}
$$

where $\boldsymbol{k}_{\|}=\left(k_{x}, k_{y}, 0\right)$. In Eq. (2), we assume periodic boundary conditions in order to accommodate the infinite dimensions in the $x$ and $y$ directions. The lateral dimensions $L_{x}$ and $L_{y}$ are normalization factors and do not affect the conductance spectrum. The Hamiltonian becomes

$$
\check{\mathcal{H}}_{\boldsymbol{k}_{\|}}(z, H)=\left[\begin{array}{cc}
\hat{h}_{\boldsymbol{k}_{\|}}(z, H) & \hat{\Delta}_{\boldsymbol{k}_{\|}}(z) \\
-\hat{\Delta}_{-\boldsymbol{k}_{\|}}^{*}(z) & -\hat{h}_{-\boldsymbol{k}_{\|}}^{*}(z, H)
\end{array}\right] .
$$

The single-particle Hamiltonian $\hat{h}_{\boldsymbol{k}_{\|}}$is given by

$$
\begin{gathered}
\hat{h}_{\boldsymbol{k}_{\|}}(z, H)=\xi_{\boldsymbol{k}_{\|}}(z, H)+\boldsymbol{M}(z) \cdot \hat{\boldsymbol{\sigma}}+\hat{F}_{\boldsymbol{k}_{\|}}(z), \\
\xi_{\boldsymbol{k}_{\|}}(z, H)=-\frac{\hbar^{2}}{2 m_{z}} \frac{\partial^{2}}{\partial z^{2}}-\mu^{\prime}-\Delta_{0} \frac{H}{H_{c}} \frac{k_{\|}}{k_{F}} \sin \varphi, \\
\mu^{\prime}=\mu-\frac{\hbar^{2}}{2}\left[\frac{k_{x}^{2}}{m_{x}}+\frac{k_{y}^{2}}{m_{y}}\right],
\end{gathered}
$$

where $\xi_{\boldsymbol{k}_{\|}}$is the kinetic energy in the presence of an external magnetic field in the $x$ direction and $\mu$ is the chemical potential, which we assume to be constant across the junction. A full derivation of Eq. (6) is given in Appendix A. The matrices $\hat{\sigma}_{j}(j \in\{x, y, z\})$ and $\hat{\sigma}_{0}$ are the Pauli matrices and the identity matrix in spin space, $\hat{\boldsymbol{\sigma}}=\hat{\sigma}_{x} \boldsymbol{e}_{x}+\hat{\sigma}_{y} \boldsymbol{e}_{y}+\hat{\sigma}_{z} \boldsymbol{e}_{z}$, with $\boldsymbol{e}_{j}$ being the unit vectors in the $j$ direction. We can modify the shape of Fermi surfaces by tuning the effective masses $\boldsymbol{m}=\left(m_{x}, m_{y}, m_{z}\right)$ in each region. In this paper, we parametrize $\boldsymbol{m}$ as

$$
\boldsymbol{m}(z)= \begin{cases}\left(m_{N}, m_{N}, m_{N}\right) & \text { for } \quad z \leqslant 0, \\ \left(m_{F}, m_{F}, m_{F}\right) & \text { for } \quad 0<z<L, \\ \left(m_{\|}, m_{\|}, m_{\perp}\right) & \text { for } \quad z \geqslant L .\end{cases}
$$

The magnetization is described as [60]

$$
\boldsymbol{M}(z)=M_{0}\left(\sin \theta_{M} \boldsymbol{e}_{x}+\cos \theta_{M} \boldsymbol{e}_{z}\right) \Theta(z) \Theta(L-z),
$$

where $\Theta(z)$ is the Heaviside step function. In this paper, we ignore the reorientation of the $d$ vector by the magnetization in F [62-65] for simplicity. The effects of the interfaces are described by $\hat{F}_{\boldsymbol{k}_{\|}}(z)$ as [66]

$$
\begin{gathered}
\hat{F}_{\boldsymbol{k}_{\|}}(z)=\delta(z) \hat{F}_{1}+\delta(z-L) \hat{F}_{2}, \\
\hat{F}_{1, \boldsymbol{k}_{\|}}=F_{1} \hat{\sigma}_{0}, \\
\hat{F}_{2, \boldsymbol{k}_{\|}}=F_{\mathrm{SO}} \boldsymbol{e}_{z} \cdot(\hat{\boldsymbol{\sigma}} \times \boldsymbol{k}),
\end{gathered}
$$

where $F_{1}$ and $F_{\text {SO }}$ represent the strengths of the barrier potential at $z=0$ and the spin-orbit coupling (SOC) at $z=L$, respectively. The SOC term reduces to

$$
\begin{aligned}
\boldsymbol{e}_{z} \cdot(\hat{\boldsymbol{\sigma}} \times \boldsymbol{k}) & =\hat{\sigma}_{x} k_{y}-\hat{\sigma}_{y} k_{x} \\
& =i k_{\|}\left[\begin{array}{cc}
0 & e^{-i \varphi} \\
-e^{+i \varphi} & 0
\end{array}\right],
\end{aligned}
$$

where $k_{x}=k_{\|} \cos \varphi$ and $k_{x}=k_{\|} \sin \varphi$, with $k_{\|}=\left(k_{x}^{2}+k_{y}^{2}\right)^{1 / 2}$. The pair potential is described by

$$
\hat{\Delta}_{\boldsymbol{k}_{\|}}(z)=\underline{\hat{\hat{\Delta}}}_{\boldsymbol{k}_{\|}}(z) \Theta(z-L) .
$$

The momentum dependences of the pair potentials for $s$-wave (SW), chiral $p$-wave (CPW), and helical $p$-wave (HPW) superconductors are written as

$$
\underline{\hat{\Delta}}_{\boldsymbol{k}_{\|}}(z)= \begin{cases}\Delta_{0} i \hat{\sigma}_{y} & \text { for SW, } \\ \Delta_{0}\left[\bar{k}_{x}+i \chi \bar{k}_{y}\right] \hat{\sigma}_{x} & \text { for CPW, } \\ \Delta_{0}\left[\bar{k}_{x} \hat{\sigma}_{0}+i \bar{k}_{y} \hat{\sigma}_{z}\right] & \text { for HPW, }\end{cases}
$$

where $\Delta_{0}$ is a constant which characterizes the amplitude of the pair potential, $\chi$ is the so-called chirality (which can be \pm 1 ), and $\bar{k}_{x}=k_{x} / k_{s \|}$, with $k_{s \|}=\sqrt{2 m_{\|} \mu^{\prime}} / \hbar$ being the Fermi wave number in the $k_{x}-k_{y}$ plane for $\mathrm{S}$. The assumption that $\Delta_{0}$ is constant implies that we do not take the inverse proximity effect (from $\mathrm{F}$ into $\mathrm{S}$ ) into account, which is a common assumption [67].

\section{B. Wave functions}

The wave function is obtained by solving the Hamiltonian at an energy $E$ in each region. Throughout this paper, we assume $E \sim \Delta_{0} \ll \mu$. The wave function for $z \leqslant 0$ is given by

$$
\Psi_{k_{\|}}(z)=\check{K}_{N}^{+} \vec{i}+\check{K}_{N}^{-} \vec{r},
$$

where $\check{K}_{N}^{ \pm}=e^{ \pm i \check{\tau}_{z} k_{N} z}$, with $k_{N}=\sqrt{2 m_{N} \mu^{\prime}} / \hbar$ and $\check{\tau}_{z}=$ $\operatorname{diag}\left[\hat{\sigma}_{0},-\hat{\sigma}_{0}\right]$ being the third Pauli matrix in Nambu space. The vector $\vec{i}$ represents the wave function amplitude of the 
incident particles, which is given by

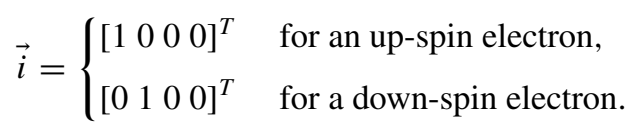

The vector $\vec{r}$ describes the wave function amplitude of the reflected particles as

$$
\vec{r}=\left[r_{\uparrow}^{p} r_{\downarrow}^{p} r_{\uparrow}^{h} r_{\downarrow}^{h}\right]^{T},
$$

where $r_{\alpha}^{p}$ and $r_{\alpha}^{h}$, with $\alpha \in\{\uparrow, \downarrow\}$, are the normal and Andreev reflection coefficients, respectively. The wave function for $0<z<L$ is given by

$$
\Psi_{\boldsymbol{k}_{\|}}(z)=\check{A} \check{K}_{F}^{+} \vec{f}_{P}+\check{A} \check{K}_{F}^{-} \vec{f}_{N},
$$

where $\check{K}_{F}^{ \pm}=\operatorname{diag}\left[e^{ \pm i k_{F}^{+} z}, e^{ \pm i k_{F}^{-} z}, e^{ \pm i k_{F}^{+} z}, e^{ \pm i k_{F}^{-} z}\right]$, with $k_{F}^{ \pm}=$ $\sqrt{2 m_{F}\left(\mu^{\prime} \mp M_{0}\right)} / \hbar$. The matrix $\check{A}=\operatorname{diag}[\hat{A}, \hat{A}]$ characterizes the spin structure of the F, where $\hat{A}$ is given by [60]

$$
\hat{A}=\left[\begin{array}{rr}
\cos \left(\theta_{M} / 2\right) & -\sin \left(\theta_{M} / 2\right) \\
\sin \left(\theta_{M} / 2\right) & \cos \left(\theta_{M} / 2\right)
\end{array}\right] .
$$

The vectors $\vec{f}_{P(N)}$ describe the wave function amplitudes of particles propagating in the positive (negative) $z$ direction. They are defined as

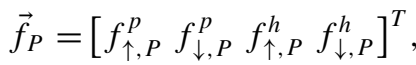

$$
\begin{aligned}
& \vec{f}_{N}=\left[f_{\uparrow, N}^{p} f_{\downarrow, N}^{p} f_{\uparrow, N}^{h} f_{\downarrow, N}^{h}\right]^{T} .
\end{aligned}
$$

The wave function for $z \geqslant L$ is given by

$$
\Psi_{k_{\|}}(z)=\check{U} \check{K}_{S} \vec{t},
$$

where $\check{K}_{S}=\operatorname{diag}\left[e^{+i k_{s} z}, e^{+i k_{S} z}, e^{-i k_{s} z}, e^{-i k_{s} z}\right]$, with $k_{S}=$ $\sqrt{2 m_{\perp} \mu^{\prime}} / \hbar$. The vector $\vec{t}$ describes the wave function amplitudes of the transmitted particles as

$$
\vec{t}=\left[t_{\uparrow}^{p} t_{\downarrow}^{p} t_{\uparrow}^{h} t_{\downarrow}^{h}\right]^{T} .
$$

The matrix $\breve{U}$ describes the amplitude of the wave function in the superconductor as

$$
\begin{gathered}
\check{U}=\left[\begin{array}{ll}
u_{\boldsymbol{k}_{\|}} \hat{\sigma}_{0} & v_{\boldsymbol{k}_{\|}} \hat{D}_{\boldsymbol{k}_{\|}} \\
v_{\boldsymbol{k}_{\|}} \hat{D}_{\boldsymbol{k}_{\|}}^{\dagger} & u_{\boldsymbol{k}_{\|}} \hat{\sigma}_{0}
\end{array}\right], \\
\hat{D}_{\boldsymbol{k}_{\|}}=\underline{\hat{\Delta}}_{\boldsymbol{k}_{\|}} / \Delta_{0},
\end{gathered}
$$

with

$$
\begin{aligned}
& u_{k_{\|}}=\frac{1}{\sqrt{2}} \sqrt{1+\frac{\Omega_{k_{\|}}}{E}}, \\
& v_{k_{\|}}=\frac{1}{\sqrt{2}} \sqrt{1-\frac{\Omega_{k_{\|}}}{E}}, \\
& \Omega_{k_{\|}}=\sqrt{E^{2}-\left|d_{k_{\|}}\right|^{2}},
\end{aligned}
$$

where $d_{\boldsymbol{k}_{\|}}$is obtained from the relation $d_{\boldsymbol{k}_{\|}} \hat{\sigma}_{0}=\underline{\hat{\hat{S}}}_{\boldsymbol{k}_{\|}} \hat{\underline{\hat{\Delta}}}_{\boldsymbol{k}_{\|}}^{\dagger}$.

\section{Differential conductance}

All coefficients in Eqs. (17), (20), and (24) can be determined by four boundary conditions at $z=0$ and $z=L$. The first two boundary conditions are derived from continuity at $z=0$. They are given by [67]

$$
\begin{gathered}
\lim _{z \uparrow 0} \Psi_{\boldsymbol{k}_{\|}}=\lim _{z \downarrow 0} \Psi_{\boldsymbol{k}_{\|}}, \\
\lim _{z \uparrow 0}\left[\frac{\partial \Psi_{\boldsymbol{k}_{\|}}}{\partial z}+\frac{2 m(z)}{\hbar^{2}} \breve{F}_{1} \Psi_{\boldsymbol{k}_{\|}}\right]=\lim _{z \downarrow 0} \frac{\partial \Psi_{\boldsymbol{k}_{\|}}}{\partial z},
\end{gathered}
$$

where $\breve{F}_{1}=\operatorname{diag}\left[\hat{F}_{1, \boldsymbol{k}_{\|}},-\hat{F}_{1,-\boldsymbol{k}_{\|}}^{*}\right]$. The other boundary conditions are related to the interface at $z=L$ as follows:

$$
\begin{gathered}
\lim _{z \uparrow L} \Psi_{\boldsymbol{k}_{\|}}=\lim _{z \downarrow L} \Psi_{\boldsymbol{k}_{\|}}, \\
\lim _{z \uparrow L}\left[\frac{\partial \Psi_{\boldsymbol{k}_{\|}}}{\partial z}+\frac{2 m(z)}{\hbar^{2}} \breve{F}_{2} \Psi_{\boldsymbol{k}_{\|}}\right]=\lim _{z \downarrow L} \frac{\partial \Psi_{\boldsymbol{k}_{\|}}}{\partial z},
\end{gathered}
$$

where $\check{F}_{2}=\operatorname{diag}\left[\hat{F}_{2, \boldsymbol{k}_{\|}},-\hat{F}_{2,-\boldsymbol{k}_{\|}}^{*}\right]$.

In the Supplemental Material [68], we derive the expression for the current through the N/F/S junction and find that it is the same as in the original Blonder-Tinkham-Klapwijk (BTK) theory [67]. Hence, we can use the same differential tunneling conductance resulting from a spin- $\alpha$ incident particle, which is given by

$$
\begin{gathered}
\sigma(E)=\sum_{\boldsymbol{k}_{\|}, \alpha}^{\prime} \sigma_{\alpha}\left(E, \boldsymbol{k}_{\|}\right), \\
\sigma_{\alpha}\left(E, \boldsymbol{k}_{\|}\right)=1+\left|r_{\uparrow}^{h}\right|^{2}+\left|r_{\downarrow}^{h}\right|^{2}-\left|r_{\uparrow}^{p}\right|^{2}-\left|r_{\downarrow}^{p}\right|^{2},
\end{gathered}
$$

where $\sigma_{\alpha}\left(E, \boldsymbol{k}_{\|}\right)$is the angle-resolved differential conductance for a spin- $\alpha$ incident particle with $\alpha \in\{\uparrow, \downarrow\}$ [69]. To model a cylindrical Fermi surface in a quasi-two-dimensional material, we introduce a cutoff in the summation with respect to $\boldsymbol{k}_{\|}$as

$$
\sum_{\boldsymbol{k}_{\|}, \alpha}^{\prime} \cdots \equiv \sum_{\boldsymbol{k}_{\|}, \alpha} \cdots \Theta\left(\left|\boldsymbol{k}_{\|}\right|-k_{c}\right)
$$

where $k_{c}=k_{N} \sin \theta_{c}$ and $\theta_{c}$ is the cutoff angle.

\section{RESULTS}

The aim of this paper is to model the conductance of a $\mathrm{Au} / \mathrm{SrRuO}_{3} / \mathrm{Sr}_{2} \mathrm{RuO}_{4}$ junction. A realistic effective mass for the ferromagnet $\mathrm{SrRuO}_{3}$ is $m_{F}=7 m_{N}$ [70]. We approximate the $\mathrm{Sr}_{2} \mathrm{RuO}_{4} \gamma$ band by modeling the Fermi surface as an ellip$\operatorname{soid}\left(m_{\|}=1.3, m_{\perp}=16\right)$ with its top and bottom cut off $\left(\theta_{c}=\right.$ $\pi / 10)$. The chirality is set to $\chi=1$. We will compare a $N / F / S$ junction without barriers to a N/F/S junction with a small tunnel barrier $F_{1}$ at the N/F interface. Because of epitaxial growth and minimal lattice mismatch, a smooth $\mathrm{F} / \mathrm{S}$ interface is expected, and therefore, no barrier is introduced. The spin-orbit coupling is set to zero. Effects of $F_{\mathrm{SO}}$ are discussed in Appendix C.

\section{A. Direction of the magnetization}

We first show the differential conductances of a junction with a spin-singlet $s$-wave superconductor in Fig. 2(a), where results with and without the interface barrier are indicated by solid and dashed lines, respectively. Throughout this paper, the differential conductance is normalized by its value in the normal state (i.e., $\Delta_{0}=0$ ), and the energy is normalized by the maximum amplitude of the pair potential in the absence of an external magnetic field $\Delta_{0}$. As shown in Fig. 2(a), 

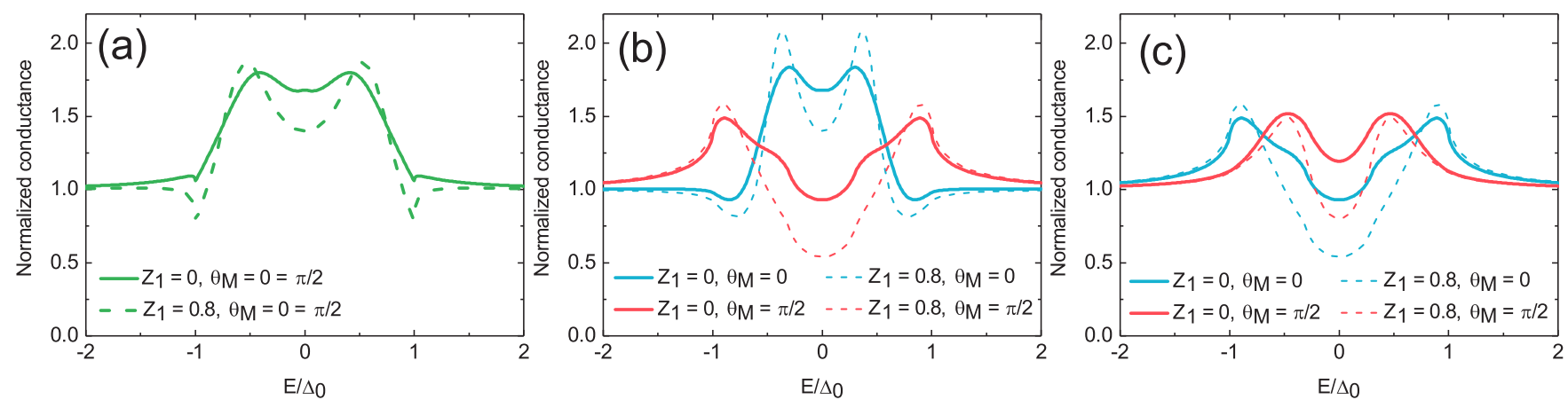

FIG. 2. The dimensionless tunneling conductance using pair potentials (a) SW, (b) CPW, and (c) HPW without barriers $\left(Z_{1}=0\right.$, solid lines) and including a small barrier at the first interface $\left(Z_{1}=0.8\right.$, dashed lines). The SW case is independent of the magnetization angle. For CPW and HPW, the magnetization angle varies from $\theta_{M}=0$ (blue lines) to $\theta_{M}=\pi / 2$ (red lines). $X=0.6, k_{F} L=11$.

the coherence peaks appear at an energy lower than the gap amplitude $\left(E \approx 0.6 \Delta_{0}\right)$, which is a result of the ferromagnet with finite thickness $L$. Comparing the solid and dashed lines, we see that the barrier potential at the N/F interface sharpens the peaks around $E \approx 0.5 \Delta_{0}$ and the dips around $E \approx \Delta_{0}$ in the differential conductance. In addition, the zero-energy dip becomes more prominent with increasing barrier strength. This is consistent with the well-known N/S junction [67]. In spin-singlet superconductors the conductance does not depend on the direction of the magnetization (i.e., $\theta_{M}$ ) because a singlet Cooper pair does not have a finite total spin. It should be noted that, throughout this paper, the pair potential is taken to be
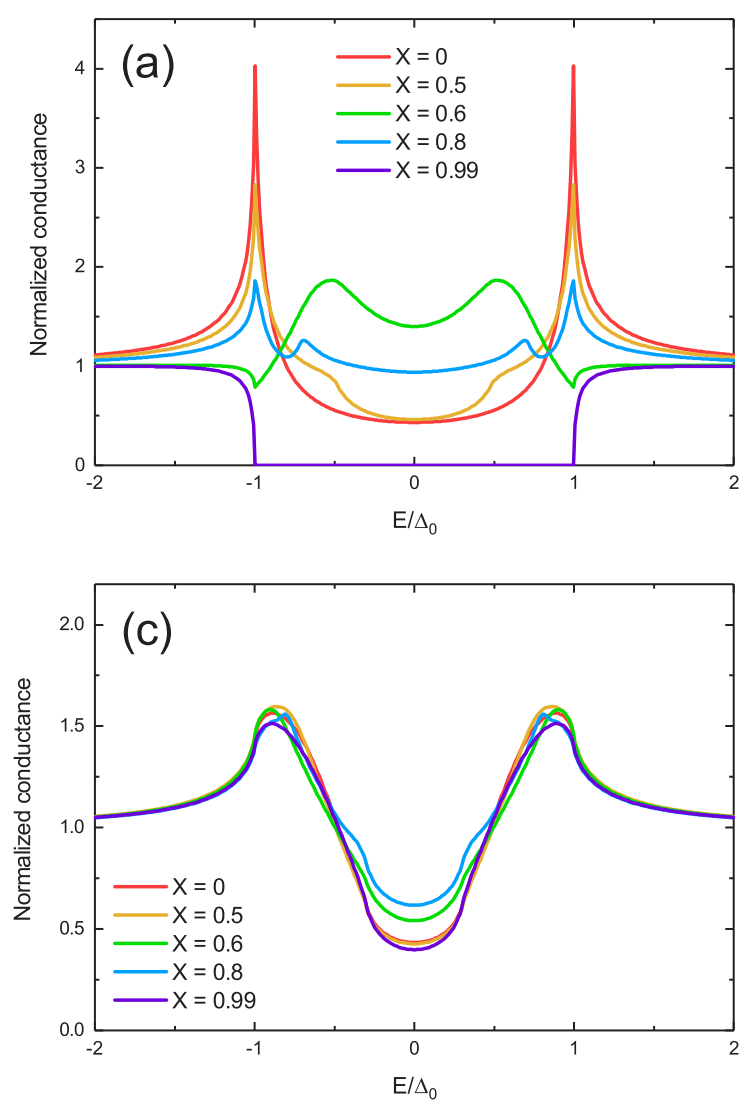

non-self-consistent (i.e., $\Delta_{0}$ is constant). The sharp peaks in the conductance would be broadened and lowered if we were to include the self-consistency [71].

The differential conductances of the spin-triplet CPW and HPW superconductors are shown in Figs. 2(b) and 2(c), respectively. The blue and red lines represent the results for $\theta_{M}=0$ and $\pi / 2$, respectively. The cases with and without the $\mathrm{N} / \mathrm{F}$ interface barrier are indicated by the solid and dashed lines, respectively. The results of the CPW with $\theta_{M}=0$ case are similar to the SW case; there are two peaks around $E \approx 0.6 \Delta_{0}$ and a dip at zero energy. The position of the peaks is determined by the $\mathrm{F}$ thickness $L$ and the magnitude of the magnetization
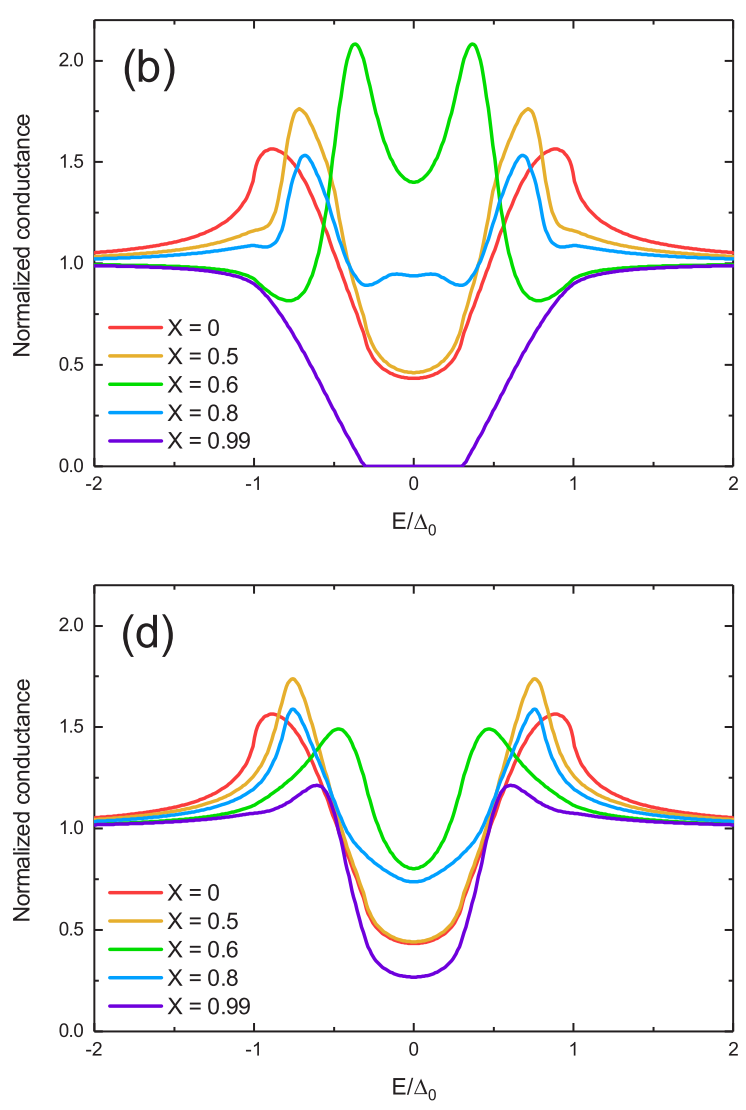

FIG. 3. Dimensionless tunneling conductance using pair potentials (a) $\mathrm{SW}$, (b) CPW with $\theta_{M}=0$, (c) HPW with $\theta_{M}=0$, which is identical to CPW with $\theta_{M}=\pi / 2$, and (d) HPW with $\theta_{M}=\pi / 2$. Magnetization strengths vary from $X=0$ (normal metal) to $X=0.99$ (fully polarized). $Z_{1}=0.8, k_{F} L=11$. 
TABLE I. Matrix structure of the pair potential. The spinquantization axis is taken to be parallel to the magnetization vector $\boldsymbol{M}$. The first and second rows are for $\boldsymbol{M} \| \boldsymbol{z}$ (i.e., $\theta_{M}=0$ ) and for $\boldsymbol{M} \| \boldsymbol{x}$ (i.e., $\theta_{M}=\pi / 2$ ), respectively. We can see that the $4 \times 4$ Hamiltonian can be reduced to two $2 \times 2$ Hamiltonian matrices except for the case of the helical $p$ wave with $\theta_{M}=\pi / 2$. The angle $\phi$ satisfies the relations $k_{x}=k_{\|} \cos \phi$ and $k_{y}=k_{\|} \sin \phi$, with $k_{\|}=\left|\boldsymbol{k}_{\|}\right|$being the momentum parallel to the interfaces. The momentum is normalized: $\bar{k}_{x(y)}=k_{x(y)} / k_{\|}$. The factor $\chi$ is the chirality of a chiral $p$-wave superconductor.

\begin{tabular}{llll}
\hline \hline & $s$-wave & Chiral $p$-wave & Helical $p$-wave \\
\hline $\boldsymbol{M} \| \boldsymbol{z}$ & {$\left[\begin{array}{rr} & 1 \\
-1 & \end{array}\right]$} & {$\left[\begin{array}{ll}e^{i \chi \phi} & e^{i \chi \phi}\end{array}\right]$} & {$\left[\begin{array}{ll}e^{i \phi} & \\
& e^{-i \phi}\end{array}\right]$} \\
$\boldsymbol{M} \| \boldsymbol{x}$ & {$\left[\begin{array}{rr} & 1 \\
-1 & \end{array}\right]$} & {$\left[\begin{array}{ll}e^{i \chi \phi} & \\
& -e^{i \chi \phi}\end{array}\right]$} & {$\left[\begin{array}{cc}\bar{k}_{x} & -i \bar{k}_{y} \\
-i \bar{k}_{y} & \bar{k}_{x}\end{array}\right]$} \\
\hline \hline
\end{tabular}

$(X \equiv M / \mu)$. In the CPW case, the Hamiltonian becomes equivalent to that for the SW case, except for the amplitude of the pair potential. Therefore, the corresponding results are qualitatively the same.

In the present case, the experimentally observed zerobias conductance peak (ZBCP) [22,23] does not appear. The Andreev bound states in CPW and HPW superconductors are located in the $b-c$ and $c-a$ planes. The junction under consideration is, however, along the $c$ axis, implying that these Andreev bound states cannot contribute to the differential conductance [72].

Comparing the red line in Fig. 2(b) to the blue line in Fig. 2(c), we find that the conductance spectra of CPW with $\theta_{M}=\pi / 2$ and HPW with $\theta_{M}=0$ are identical. In both cases, the $d$ vector is perpendicular to the magnetization $(\boldsymbol{d} \perp \boldsymbol{M})$; that is, the total spin of the Cooper pairs is parallel to the magnetization.

By analytically rotating the spin quantization axis, we reduce the matrix form of the pair potential matrix in the proper spin axis in which the $z$ direction is parallel to the magnetization. By doing this, we demonstrate that the pair potentials in the cases of CPW with $\theta_{M}=\pi / 2$ and HPW with $\theta_{M}=0$ are qualitatively the same, except for the spin-dependent chirality. A full derivation is given in Appendix C; the matrix structures of the pair potential are summarized in Table I. Hence, as long as there is no perturbation which mixes the spins or depends on the chirality (e.g., spin-active interface, spin-orbit coupling, or a perturbation which breaks translational symmetry in the $x$ and/or $y$ direction such as walls and impurities), it is impossible to distinguish between these two cases.

\section{B. Amplitude of the magnetization}

The effects of the amplitude of the magnetization are shown in Fig. 3, where the pair potential and the direction of the magnetization are set to SW with $\theta_{M}=0$ [Fig. 3(a)], CPW with $\theta_{M}=0$ [Fig. 3(b)], CPW with $\theta_{M}=\pi / 2$ [Fig. 3(c)], and HPW
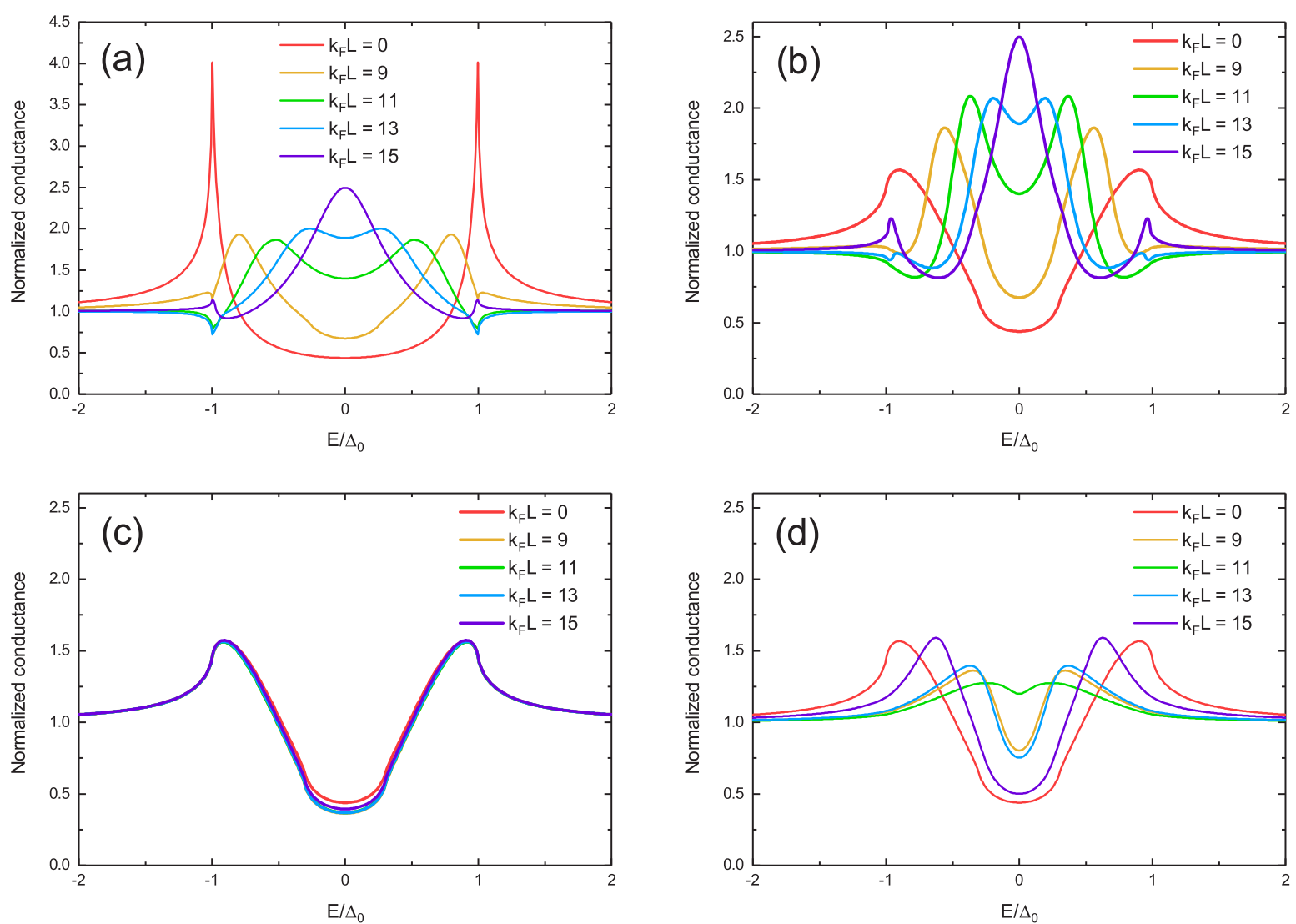

FIG. 4. The dimensionless tunneling conductance using pair potentials (a) SW, (b) CPW with $\theta_{M}=0$, (c) HPW with $\theta_{M}=0$, which is identical to CPW with $\theta_{M}=\pi / 2$, and (d) HPW with $\theta_{M}=\pi / 2$ without a ferromagnet $\left(k_{F} L=0\right)$ and for varying thicknesses $k_{F} L$ of the ferromagnet. $Z_{1}=0.8, X=0.6$. 

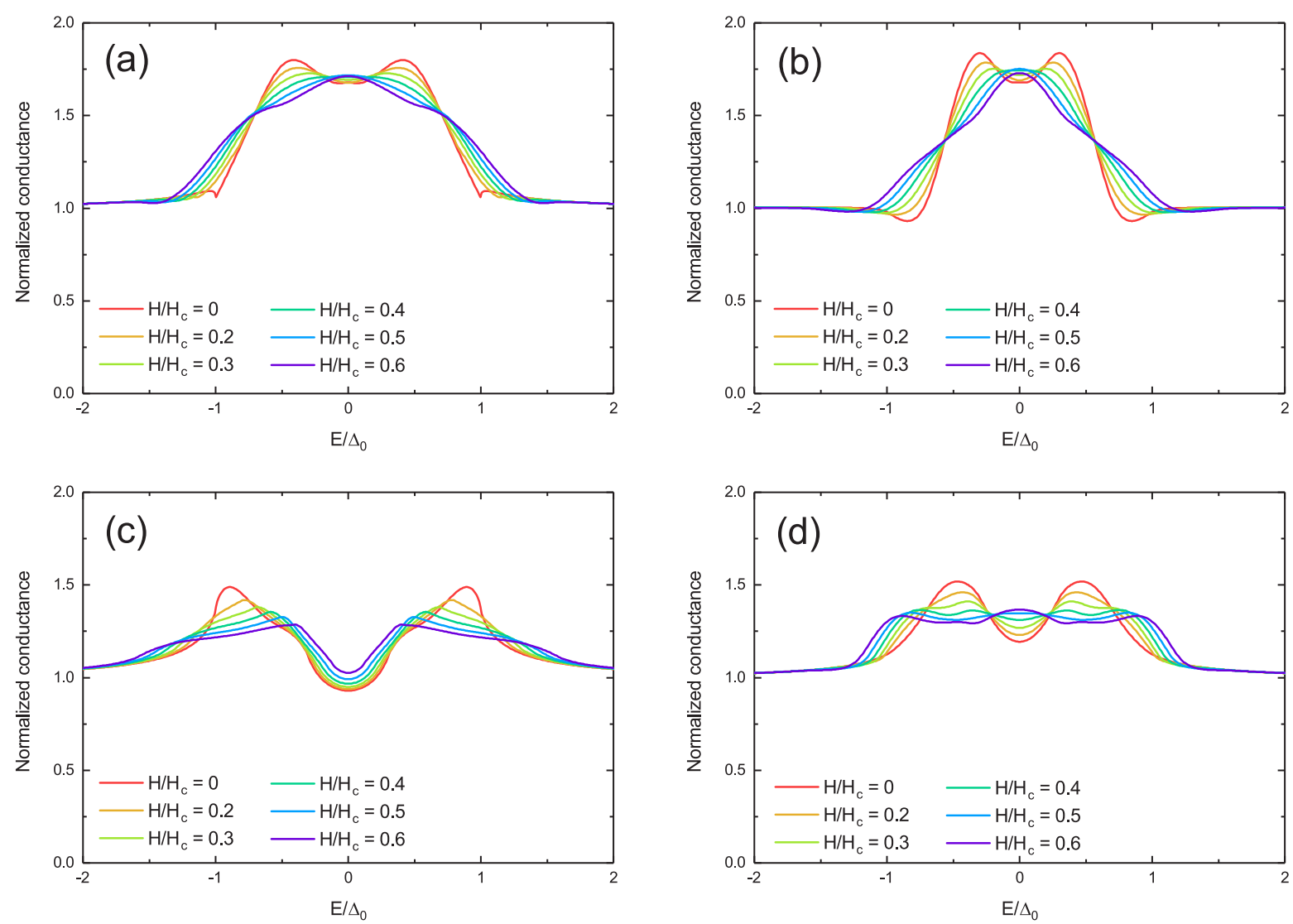

FIG. 5. Effects of an external magnetic field on the dimensionless tunneling conductance in the absence of the barrier potential. The pair potential is assumed to be (a) SW, (b) CPW with $\theta_{M}=0$, (c) HPW with $\theta_{M}=0$, and (d) HPW with $\theta_{M}=\pi / 2$. The results for the CPW with $\theta_{M}=\pi / 2$ are identical to the results in (c). The parameters are set to $Z_{1}=0, X=0.6$, and $k_{F} L=11$.

with $\theta_{M}=\pi / 2$ [Fig. 3(d)]. We note that the result for the CPW with $\theta_{M}=\pi / 2$ and that for the HPW with $\theta_{M}=0$ are identical to each other. The barrier strength and the thickness of the ferromagnet are set to $Z_{1}=0.8$ and $k_{F} L=11$, respectively.

In the SW case in the absence of magnetization $(X=0)$, we obtain the BTK-like U-shaped spectrum [67], as shown in Fig. 3(a). Since the system is regarded as a N/N/S junction when $X=0$, this result is well understood within the BTK theory. When the ferromagnet is fully spin polarized $(X \approx 1)$, the conductance becomes zero in the energy range $|E|<\Delta_{0}$. Since there is no propagating channel in the $\mathrm{S}$, a quasiparticle with energy $|E|<\Delta_{0}$ must be either normally or Andreev reflected at the F/S interface. In spin-singlet superconductors, Andreev reflection is always accompanied by a spin flip (e.g., an up-spin particle is reflected as a down-spin hole). On the other hand, there is only one band in a fully polarized ferromagnet, which implies that Andreev reflection is prohibited. As a result, the conductance in the energy range $|E|<\Delta_{0}$ is always zero. For moderate spin polarizations, the conductance spectra have complex structures that are sensitive to the amplitude of $\boldsymbol{M}$.

The conductance spectrum in the CPW with $\theta_{M}=0$ case [Fig. 3(b)] is qualitatively the same as the SW spectrum because Cooper pairs consist of quasiparticles with opposite spins. However, the CPW conductance changes more gradually as a function of magnetization because the amplitude of the pair potential changes depending on $k_{z}$. In the cases where $\boldsymbol{d} \perp \boldsymbol{M}$ [Fig. 3(c)], the conductance spectra do not depend on $\boldsymbol{M}$ qualitatively because the total spin of the Cooper pairs aligns with the magnetization. This implies that the presence of the ferromagnet does not affect the superconductivity, and therefore, the conductance spectra are insensitive to the magnetization. Contrary to Figs. 3(a) and 3(b), the conductance in the HPW with $\theta_{M}=\pi / 2$ case [Fig. 3(d)] remains finite even if $X \approx 1$. In HPW superconductors, the $d$ vector lies in the $x y$ plain in spin space. Therefore, the $\boldsymbol{k}_{\|}$-dependent part of the Andreev reflection is suppressed by the magnetization in the $x$ direction.

\section{Thickness of the ferromagnet}

In Fig. 4, the conductance spectra are plotted for several thicknesses of the ferromagnetic layer $L$. In the SW junction [Fig. 4(a)], the conductance shows the BTK-like U-shaped spectrum [67], as seen in Fig. 3(a) with $X=0$. The distance between the two peaks decreases with increasing thickness. Simultaneously, the structures at $E=\Delta_{0}$ change from peaks to dips. When $k_{F} L=15$, the two peaks merge into a ZBCP. We note that this peak is different from the well-known ZBCP in $d$-wave superconductors, which stems from the interference between incident and reflected quasiparticles at the interface. On the other hand, the peak at the zero energy in Fig. 3(a) is formed by an accidental constructive Fabry-Pérot interference in the ferromagnet [73]. Hence, this peak is not robustly 

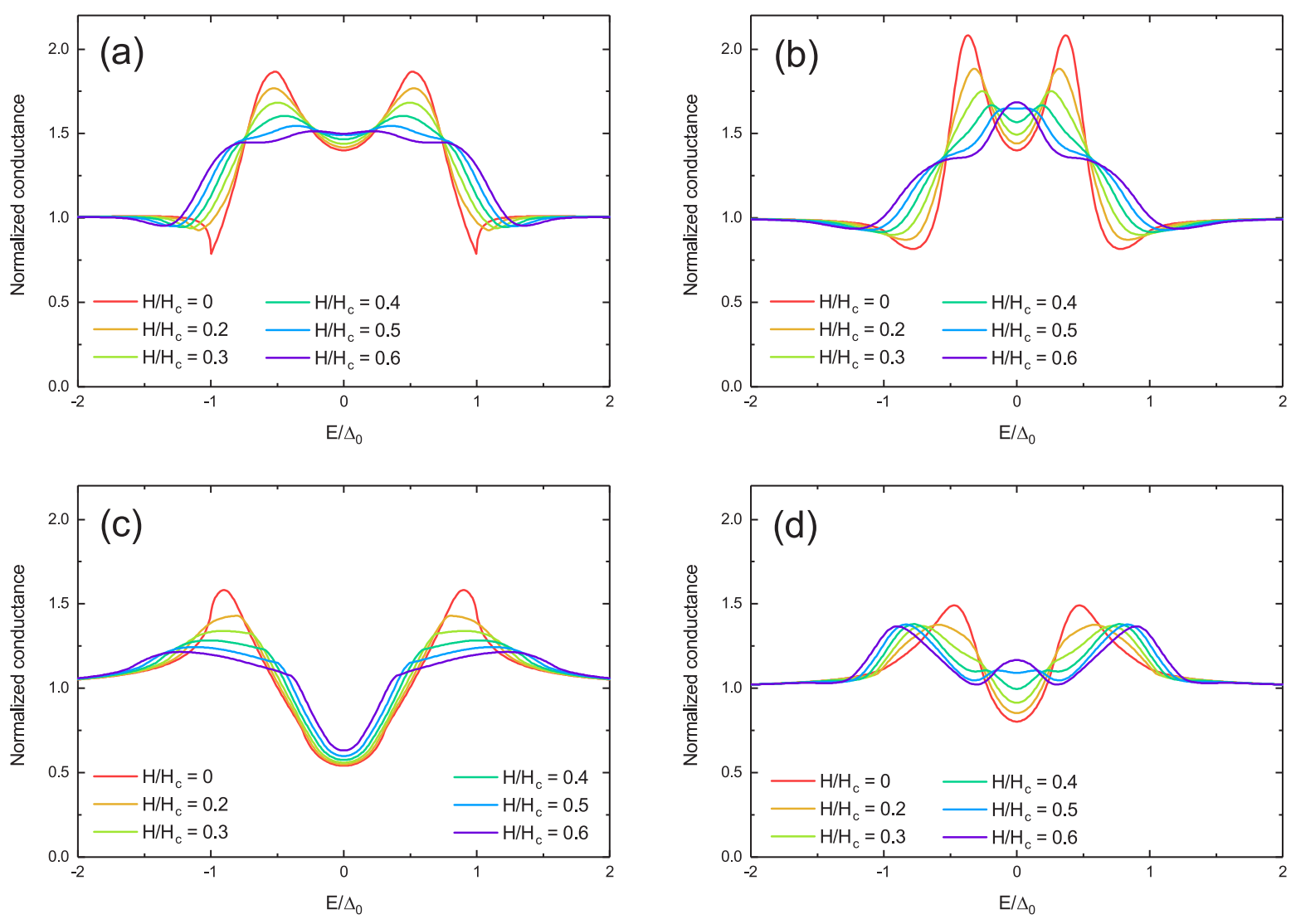

FIG. 6. Effects of an external magnetic field on the dimensionless tunneling conductance in the presence of the barrier potential $Z_{1}=0.8$ in the same manner as in Fig. 5.

resistant to impurities and is therefore not related to the topology in the superconductor.

Similar behavior is seen in the spectrum of the CPW with $\theta_{M}=0$ case [Fig. 4(b)]. In HPW superconductors [Fig. 4(d)], the distance between the two peaks first reduces for $0 \leqslant k_{F} L \leqslant$ 11 , whereas it increases for $11 \leqslant k_{F} L \leqslant 15$. However, the constructive interference as seen in CPW superconductors never occurs at zero energy. This is a significant difference between CPW and HPW superconductors.

When the $d$ vector is perpendicular to the magnetization (i.e., $\boldsymbol{d} \perp \boldsymbol{M}$ ), the results are insensitive to the ferromagnet thickness, as shown in Fig. 4(c). This can also be interpreted in terms of the relation between the direction of $\boldsymbol{M}$ and the total spin of Cooper pairs in the superconductor.

\section{External magnetic field}

The magnetic field dependence of the conductance in the absence (presence) of a barrier at the N/F interface is shown in Fig. 5 (Fig. 6), where the other parameters are set to the same values used in Fig. 3. The pair potential is assumed to be SW [Figs. 5(a) and 6(a)], CPW with $\theta_{M}=0$ [Figs. 5(b) and 6(b)], CPW with $\theta_{M}=\pi / 2$ [Figs. 5(c) and 6(c)], and HPW with $\theta_{M}=\pi / 2$ [Figs. 5(d) and 6(d)], where the results for the HPW with $\theta_{M}=0$ are identical to the results in Figs. 5(c) and 6(c). We show only the results for an external field $H \leqslant 0.6 H_{c}$ since the effects of the nucleation of vortices are not taken into account.
In general, the Doppler shift causes peaks to split into two smaller peaks, which shift with $k_{\|}$, as follows from Eq. (A7). Since pairing symmetries have different $k_{\|}$dependences, the evolution of the peak shape is different in each case. Both SW and CPW with $\theta_{M}=0$ [Figs. 5(a) and 5(b)] show a three-dip structure that gradually transitions into a broad ZBCP. For the CPW with $\theta_{M}=\pi / 2$ and HPW with $\theta_{M}=0$ cases [Fig. 5(c)], the coherence peaks are smeared out by the magnetic field, although the central dip remains. In the HPW with $\theta_{M}=\pi / 2$ case [Fig. 5(d)], the two peaks are split into four smaller peaks $\left[H / H_{c}=0.4\right.$ in Fig. 5(d)]. The outer peaks shift to away from zero energy, while the inner ones merge and form a small ZBCP.

Including a barrier in the SW, both the CPW and HPW with $\theta_{M}=0$ cases [Figs. 6(a)-6(c)] do not change behavior qualitatively, but the overall structure is more pronounced. In the HPW with $\theta_{M}=\pi / 2$ case [Fig. 6(d)], however, the spectrum changes from a plateau to a three-peak structure. The CPW with $\theta_{M}=0$ and HPW with $\theta_{M}=\pi / 2$ cases can be distinguished by looking at the relative peak height of the ZBCP.

\section{SUMMARY}

We have investigated the conductance of a N/F/S junction with various pair potentials as a function of ferromagnetic properties (thickness, magnetization strength, and direction). The SW and CPW with $\theta_{M}=0$ cases are similar, although 
the latter shows a more rounded conductance due to the angle dependence of the pair potential. We found that the cases where the $d$ vector is perpendicular to the magnetization direction (CPW with $\theta_{M}=\pi / 2$ and HPW with $\theta_{M}=0$ ) are identical. In these cases, the opposite-spin parts of the Hamiltonian are decoupled, and therefore, they are insensitive to the ferromagnet thickness and magnetization strength. The cases where the $d$ vector is parallel to the magnetization direction are very different due to a more complex structure. The main difference is that CPW with $\theta_{M}=0$ converges to a zero-energy peak for $k_{F} L=$ 15 , while HPW with $\theta_{M}=\pi / 2$ shows a dip. In the presence of an external magnetic field, the evolution of the conductance spectra depends on the pairing symmetry. In particular, the CPW with $\theta_{M}=0$ case gives an accidental ZBCP. The central dip in the CPW with $\theta_{M}=\pi / 2$ and HPW with $\theta_{M}=0$ cases remains. In the HPW with $\theta_{M}=\pi / 2$ case, the structure depends on the barrier strength: a plateau or three peaks.

For future research, it would be interesting to take higher applied magnetic fields into account by including Abrikosov vortices. To obtain a more accurate representation of $\mathrm{Sr}_{2} \mathrm{RuO}_{4}$, tunneling spectroscopy can be simulated using a multiband model $[74,75]$.

\section{ACKNOWLEDGMENTS}

This work was supported by the JSPS Core-to-Core program "Oxide Superspin" international network, a Grant-inAid for Scientific Research on Innovative Areas Topological Material Science JPSJ KAKENHI (Grants No. JP15H05851, No. 15H05852, No. JP15H05853, and No. JP15K21717), and a Grant-in-Aid for Scientific Research B (Grants No. JP15H03686 and No. JP18H01176). It was also supported by JSPS-RFBR Bilateral Joint Research Projects and Seminars Grant No. 17-52-50080, Ministry of Education and Science of the Russian Federation Grant No. 14.Y26.31.0007, and by Russian-Greek projects RFMEFI61717X0001 and T4 $\Delta \mathrm{P} \Omega$ 00031.

\section{APPENDIX A: DOPPLER SHIFT}

In the presence of a magnetic field $\boldsymbol{H}=\boldsymbol{\nabla} \times \boldsymbol{A}$ the canonical momentum operator $\boldsymbol{p}$ is replaced by the kinetic momentum operator $\boldsymbol{\pi}=\boldsymbol{p}-\boldsymbol{e} \boldsymbol{A}(\vec{r}) / c$. As a result, the quasiparticle kinetic energy $\xi_{k}$ becomes

$$
\xi_{k}=\frac{1}{2 m} \pi \cdot \pi-\mu=-\frac{\hbar^{2}}{2 m}\left(\nabla-i \frac{|e|}{\hbar c} \boldsymbol{A}\right)^{2}-\mu,
$$

where $\mu$ is the chemical potential. In the weak-coupling limit $\left(\Delta_{0} \ll \mu\right)$, this can be approximated by

$$
\xi_{k} \approx-\frac{\hbar^{2} \nabla^{2}}{2 m}-i \frac{\hbar|e|}{m c} \nabla \cdot \boldsymbol{A}-\mu
$$

In our case, an external magnetic field $\boldsymbol{H}$ is applied in the $x$ direction. Hence, the magnetic field and vector potentials for $z \geqslant 0$ are approximately [76]

$$
\begin{gathered}
\boldsymbol{H}(z)=H e^{-z / \lambda_{L}} \boldsymbol{e}_{x}, \\
\boldsymbol{A}(z)=-H \lambda_{L} e^{-z / \lambda_{L}} \boldsymbol{e}_{y},
\end{gathered}
$$

where $\lambda_{L}$ is the London penetration depth. The spatial dependence of $\boldsymbol{A}$ is characterized by $\lambda_{L}$, whereas the Cooper pair wave function is characterized by the coherence length $\xi_{0}$. In the type-II limit $\left(\lambda_{L} / \xi_{0} \gg 1\right)$, the spatial dependence of $\boldsymbol{A}$ does not change the differential conductance. Therefore, we introduce the constant vector potential [77]

$$
\boldsymbol{A}(z) \approx-H \lambda_{L} \boldsymbol{e}_{y}
$$

This linear response is valid only in the absence of vortices, i.e., for small magnetic fields $\left(H \leqslant 0.6 H_{c}\right)$. Assuming plane waves in the $x$ and $y$ directions, the wave function can be written as $\psi(x, y, z)=\psi(z) e^{i k_{x} x} e^{i k_{y} y}$, such that Eq. (A2) becomes

$$
\xi_{k}=-\frac{\hbar^{2}}{2 m} \frac{\partial^{2}}{\partial z^{2}}+\frac{\hbar^{2} k_{\|}^{2}}{2 m}-\frac{\hbar|e|}{m c} H \lambda_{L} k_{y}-\mu,
$$

where $k_{\|}^{2}=k_{x}^{2}+k_{y}^{2}$. Defining $\mu^{\prime} \equiv \mu-\hbar^{2} k_{\|}^{2} / 2 m$ and substituting $H_{c}=\phi_{0} / \pi \xi_{0} \lambda_{L}, \phi_{0}=\pi \hbar c /|e|, \xi_{0}=\hbar v_{F} / \Delta_{0}, k_{y}=$ $k_{\|} \sin \varphi$, and $v_{F}=\hbar k_{F} / m$, Eq. (A6) can be written as

$$
\xi_{k}=-\frac{\hbar^{2}}{2 m} \frac{\partial^{2}}{\partial z^{2}}-\mu^{\prime}-\Delta_{0} \frac{H}{H_{c}} \frac{k_{\|}}{k_{F}} \sin \varphi,
$$

where $H_{c}$ is the thermodynamical critical field.

\section{APPENDIX B: NUMERICAL METHOD}

Substituting wave functions (17) and (20) into boundary condition (31) gives

$$
\vec{i}+\vec{r}=\check{A}\left(\vec{f}_{p}+\vec{f}_{n}\right)
$$

We do the same with boundary condition (32) and divide by $i k_{0}$ for normalization, where we define $k_{0}$ as the momentum in the normal metal, i.e., $k_{0}=\sqrt{2 m_{N} \mu} / \hbar$. The second boundary condition becomes

$$
\left(\frac{k_{N}}{k_{0}} \check{\tau}_{0}-2 i \check{Z}_{1}\right) \vec{i}-\left(\frac{k_{N}}{k_{0}} \check{\tau}_{z}+2 i \check{Z}_{1}\right) \vec{r}=\check{A} \check{Q}\left(\vec{f}_{p}-\vec{f}_{n}\right),
$$

where $\check{Q}=\operatorname{diag}\left[k_{F}^{+}, k_{F}^{-}, k_{F}^{+}, k_{F}^{-}\right] / k_{0}$ and $\check{Z}_{1}$ is the dimensionless barrier strength of the first interface, given by

$$
\check{Z}_{1}=\frac{m(z) \check{F}_{1}}{\hbar^{2} k_{0}} \text {. }
$$

We substitute wave functions (20) and (24) into the third boundary condition, Eq. (33), to obtain

$$
\check{A} \check{K}_{F}^{L+} \vec{f}_{p}+\check{A} \check{K}_{F}^{L-} \vec{f}_{n}=\check{U} \check{K}_{S}^{L} \vec{t},
$$

where we used $\check{K}_{F}^{L \pm}=\left.\check{K}_{F}^{ \pm}\right|_{z=L}$ and $\check{K}_{S}^{L \pm}=\left.\check{K}_{S}^{ \pm}\right|_{z=L}$ for brevity. Similarly, from Eq. (34), we get

$$
\begin{aligned}
& \check{A} \check{Q}\left(\check{K}_{F}^{L+} \vec{f}_{p}-\check{K}_{F}^{L-} \vec{f}_{n}\right)-2 i \check{Z}_{\mathrm{SO}} \check{A}\left(\check{K}_{F}^{L+} \vec{f}_{p}+\check{K}_{F}^{L-} \vec{f}_{n}\right) \\
& \quad=\frac{k_{S}}{k_{0}} \check{U} \check{\tau}_{z} \check{K}_{S}^{L} \vec{t}
\end{aligned}
$$

where $\check{Z}_{\text {SO }}$ is the dimensionless spin-orbit coupling strength at the second interface, defined as

$$
\check{Z}_{\mathrm{SO}}=\frac{m(z) \check{F}_{\mathrm{SO}}}{\hbar^{2} k_{0}} .
$$

Equations (B1), (B2), (B4), and (B5) form a system of 16 equations with 16 unknowns. Substituting Eqs. (B4) and (B5) 
into one another, we can write $\check{M}_{1} \vec{f}_{p}=\check{M}_{2} \vec{f}_{n}$, with

$$
\begin{aligned}
& \check{M}_{1}=\check{A} \check{Q} \check{K}_{F}^{L+}-2 i \check{Z}_{\mathrm{SO}} \check{A} \check{K}_{F}^{L+}-\frac{k_{S}}{k_{0}} \check{U}_{\bar{\tau}} \check{\tau}_{z} \check{U}^{-1} \check{A}_{\bar{K}}^{L+}, \\
& \check{M}_{2}=\check{A} \check{Q} \check{K}_{F}^{L-}+2 i \check{Z}_{\mathrm{SO}} \check{A} \check{K}_{F}^{L-}+\frac{k_{S}}{k_{0}} \check{U}_{\bar{\tau}} \check{\tau}_{z} \check{U}^{-1} \check{A}_{\bar{K}}^{L-} .
\end{aligned}
$$

Combining this with Eq. (B2), we can express $\vec{f}_{p}$ and $\vec{f}_{n}$ in terms of $\vec{i}$ and $\vec{r}$ as

$$
\begin{gathered}
\vec{f}_{n}=\check{M}_{3}^{-1}(\vec{i}+\vec{r}), \\
\vec{f}_{p}=\check{M}_{1}^{-1} \check{M}_{2} \check{M}_{3}^{-1}(\vec{i}+\vec{r}),
\end{gathered}
$$

where $\check{M}_{3}=\check{A}\left(\check{M}_{1}^{-1} \check{M}_{2}+\check{\tau}_{0}\right)$. Substituting Eqs. (B7) and (B8) into Eq. (B1), we find that

$$
\vec{r}=\check{M}_{4}^{-1} \check{M}_{5} \vec{i},
$$

with

$$
\begin{aligned}
& \check{M}_{4}=\frac{k_{N}}{k_{0}} \check{\tau}_{z}+2 i \check{Z}_{1}-\check{A} \check{Q}\left(\check{M}_{1}^{-1} \check{M}_{2}-\vec{\sigma}_{0}\right) \check{M}_{3}^{-1}, \\
& \check{M}_{5}=\frac{k_{N}}{k_{0}} \check{\tau}_{0}-2 i \check{Z}_{1}+\check{A} \check{Q}\left(\check{M}_{1}^{-1} \check{M}_{2}-\vec{\sigma}_{0}\right) \check{M}_{3}^{-1} .
\end{aligned}
$$

Using the $\vec{r}$ coefficients, the conductance can be determined by Eq. (36).

\section{APPENDIX C: ROTATION OF THE SPIN QUANTIZATION AXIS}

To discuss the spin of Cooper pairs, it is convenient to rotate the spin quantization axis such that the new $z$ axis is parallel to the magnetization $\boldsymbol{M}$. In our case, $\boldsymbol{M}$ is in the $x z$ plane in spin space. Therefore, the rotation should be around the $y$ axis in spin space, which is carried out by the unitary operator

$$
\begin{aligned}
\hat{U}\left(\theta_{M}\right) & =\exp \left[i\left(\theta_{M} / 2\right) \hat{\sigma}_{y}\right] \\
& =\hat{\sigma}_{0} \cos \left(\theta_{M} / 2\right)+i \hat{\sigma}_{y} \sin \left(\theta_{M} / 2\right),
\end{aligned}
$$

with which we can rotate spin space by an angle $\theta_{M}$. The unitary matrix in Eq. (C2) satisfies $\hat{U}^{*}=\hat{U}$, and therefore, the unitary matrix in Nambu space is given by $\breve{U}=\operatorname{diag}\left[\hat{U}, \hat{U}^{*}\right]=$ $\operatorname{diag}[\hat{U}, \hat{U}]$. The BdG equation changes accordingly and becomes

$$
\check{H} \Psi=E \Psi \quad \rightarrow \quad \check{H}^{\prime} \tilde{\Psi}=E \tilde{\Psi},
$$

with

$$
\begin{gathered}
\tilde{\Psi}=\check{U} \Psi, \quad \check{H}^{\prime}=\check{U} \breve{H}^{\breve{U}^{\dagger}}, \\
\Psi=\left[\psi_{\uparrow} \psi_{\downarrow} \psi_{\uparrow}^{\dagger} \psi_{\downarrow}^{\dagger}\right]^{T} .
\end{gathered}
$$

Only the magnetization term depends on spin in the singleparticle Hamiltonian $\hat{h}(z)$. In the new spin basis, the magnetization terms for particles and holes are given by, respectively,

$$
\begin{gathered}
\hat{U}(\boldsymbol{M} \cdot \hat{\boldsymbol{\sigma}}) \hat{U}^{\dagger}=M \hat{\sigma}_{z}, \\
\hat{U}\left(-\boldsymbol{M} \cdot \hat{\boldsymbol{\sigma}}^{*}\right) \hat{U}^{\dagger}=-M \hat{\sigma}_{z} .
\end{gathered}
$$

The pair potential in the new spin space is

$$
\left[\begin{array}{cc} 
& \hat{\hat{\Delta}}_{\boldsymbol{k}_{\|}} \\
-\underline{\hat{\Delta}}_{-\boldsymbol{k}_{\|}}^{*} &
\end{array}\right] \rightarrow\left[\begin{array}{ll}
\left.\hat{U} \underline{\hat{U}}_{-\boldsymbol{k}_{\|}} \hat{U}^{\dagger}\right]^{*}{ }^{\dagger} &
\end{array}\right],
$$

where we used the relation $\hat{U}^{*}=\hat{U}$. The superconducting pair potential $\underline{\hat{\Delta}}_{\boldsymbol{k}}$ is transformed to

$$
\begin{aligned}
& \hat{U} \underline{\hat{\Delta}}_{k_{\|}} \hat{U}^{\dagger} \\
& \quad= \begin{cases}\Delta_{0} i \hat{\sigma}_{y} & \text { for SW, } \\
\Delta_{0}\left(\bar{k}_{x}+i \chi \bar{k}_{y}\right)\left[\cos \theta_{M} \hat{\sigma}_{x}+\sin \theta_{M} \hat{\sigma}_{z}\right] & \text { for CPW, } \\
\Delta_{0}\left(\bar{k}_{x} \hat{\sigma}_{0}+i \bar{k}_{y}\left[\cos \theta_{M} \hat{\sigma}_{z}-\sin \theta_{M} \hat{\sigma}_{x}\right]\right) & \text { for HPW. }\end{cases}
\end{aligned}
$$

If we substitute $\theta_{M}=0, \pi / 2$, these expressions reduce to the pair potentials in Table I in the main text.

We focus on CPW with $\theta_{M}=\pi / 2$ and HPW with $\theta_{M}=0$. In both cases, the magnetization $\boldsymbol{M}$ is perpendicular to the $d$ vector. In other words, $\boldsymbol{M}$ and the total spin of the Cooper pairs are collinear. Therefore, the magnetization does not destroy the Cooper pairs. The $4 \times 4$ Hamiltonian matrix can be reduced to two $2 \times 2$ matrices:

$$
\begin{aligned}
\mathcal{H} & =\frac{1}{2} \sum_{\boldsymbol{k}_{\|}} \int \tilde{\Psi}^{\dagger}(z) \check{H}_{B}(z) \tilde{\Psi}(z) d z \\
& =\frac{1}{2} \sum_{\boldsymbol{k}_{\|}} \int \sum_{\alpha= \pm 1} \tilde{\Psi}_{\alpha}^{\dagger}\left[\begin{array}{cc}
\xi+\alpha M & \Delta_{\alpha, k_{\|}} \\
-\Delta_{\alpha,-k_{\|}}^{*} & -(\xi+\alpha M)
\end{array}\right] \tilde{\Psi}_{\alpha} d z .
\end{aligned}
$$

We have introduced a new basis which depends on the spin

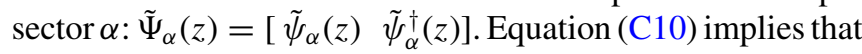
the system can be decomposed into the spin-up $(\alpha=1)$ and spin-down $(\alpha=-1)$ subsystems, where we have redefined the up and down spins for the new spin quantization axis. The

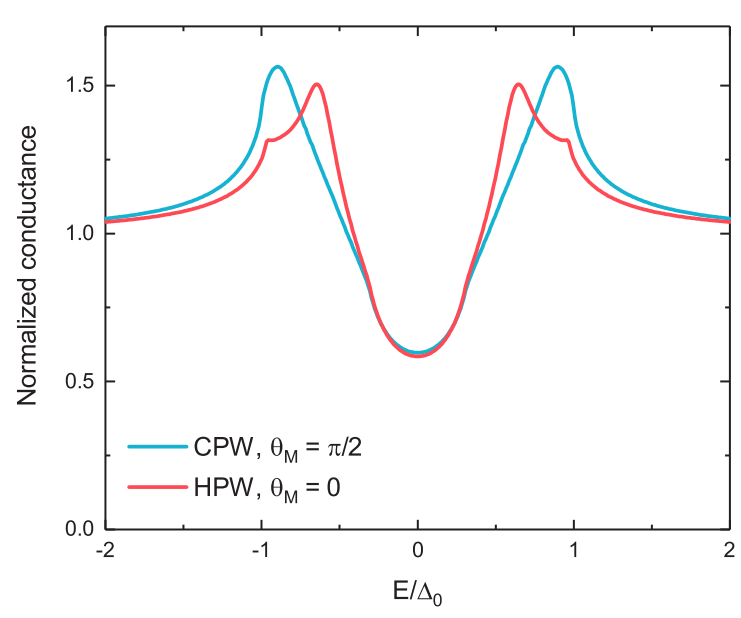

FIG. 7. The dimensionless tunneling conductance for $\mathrm{CPW}$ with $\theta_{M}=\pi / 2$ and HPW with $\theta_{M}=0$, including spin-orbit coupling $Z_{\text {SO }}=1 . Z_{1}=0.8, X=0.6, k_{F} L=11$. 
$\alpha$-dependent pair potential is given by

$$
\Delta_{\alpha, k_{\|}}=\left\{\begin{array}{lll}
\alpha \Delta_{0} e^{i \phi} & \text { for CPW } & \text { with } \theta_{M}=\pi / 2, \\
\Delta_{0} e^{i \alpha \phi} & \text { for HPW } & \text { with } \theta_{M}=0,
\end{array}\right.
$$

where we fix $\chi=1$. In the CPW with $\theta_{M}=\pi / 2$ case, the chiralities for the up- and down-spin sectors are the same, while the signs of the $\alpha$-dependent pair potential are opposite. In the HPW with $\theta_{M}=0$ case, the chiralities are opposite, while the signs of the $\alpha$-dependent pair potential are equal.
Therefore, as long as there is no perturbation which mixes the spins or depends on the chirality (e.g., spin-active interface, spin-orbit coupling, and perturbations which break translational symmetry in the $x$ and/or $y$ direction such as walls and impurities), it is impossible to distinguish these two cases.

This is demonstrated in Fig. 7, where we introduced spinorbit coupling at the $\mathrm{F} / \mathrm{S}$ interface by setting $Z_{\mathrm{SO}}=1$. In the absence spin-orbit coupling, these two graphs overlap, as seen in Figs. 3(c), 4(c), 5(c), and 6(c). However, in Fig. 7, we can see that they are, indeed, slightly different.
[1] Y. Maeno, H. Hashimoto, K. Yoshida, S. Nishizaki, T. Fujita, J. G. Bednorz, and F. Lichtenberg, Nature (London) 372, 532 (1994).

[2] K. Ishida, H. Mukuda, Y. Kitaoka, K. Asayama, Z. Q. Mao, Y. Mori, and Y. Maeno, Nature (London) 396, 658 (1998).

[3] H. Tou, Y. Kitaoka, K. Ishida, K. Asayama, N. Kimura, Y. Onuki, E. Yamamoto, Y. Haga, and K. Maezawa, Phys. Rev. Lett. 80, 3129 (1998).

[4] H. Murakawa, K. Ishida, K. Kitagawa, Z. Q. Mao, and Y. Maeno, Phys. Rev. Lett. 93, 167004 (2004).

[5] A. P. Mackenzie and Y. Maeno, Rev. Mod. Phys. 75, 657 (2003).

[6] Y. Maeno, S. Kittaka, T. Nomura, S. Yonezawa, and K. Ishida, J. Phys. Soc. Jpn. 81, 011009 (2012).

[7] T. M. Rice and M. Sigrist, J. Phys.: Condens. Matter 7, L643 (1995).

[8] K. Miyake and O. Narikiyo, Phys. Rev. Lett. 83, 1423 (1999).

[9] T. Kuwabara and M. Ogata, Phys. Rev. Lett. 85, 4586 (2000).

[10] T. Nomura and K. Yamada, J. Phys. Soc. Jpn. 69, 3678 (2000).

[11] T. Nomura and K. Yamada, J. Phys. Soc. Jpn. 71, 404 (2002).

[12] T. Nomura and K. Yamada, J. Phys. Soc. Jpn. 71, 1993 (2002).

[13] R. Arita, S. Onari, K. Kuroki, and H. Aoki, Phys. Rev. Lett. 92, 247006 (2004).

[14] T. Nomura and K. Yamada, J. Phys. Soc. Jpn. 74, 1818 (2005).

[15] T. Nomura, D. S. Hirashima, and K. Yamada, J. Phys. Soc. Jpn. 77, 024701 (2008).

[16] Y. Yanase and M. Ogata, J. Phys. Soc. Jpn. 72, 673 (2003).

[17] S. Raghu, A. Kapitulnik, and S. A. Kivelson, Phys. Rev. Lett. 105, 136401 (2010).

[18] M. Sato and M. Kohmoto, J. Phys. Soc. Jpn. 69, 3505 (2000).

[19] K. Kuroki, M. Ogata, R. Arita, and H. Aoki, Phys. Rev. B 63, 060506 (2001).

[20] T. Takimoto, Phys. Rev. B 62, R14641 (2000).

[21] M. Tsuchiizu, Y. Yamakawa, S. Onari, Y. Ohno, and H. Kontani, Phys. Rev. B 91, 155103 (2015).

[22] F. Laube, G. Goll, H. v. Löhneysen, M. Fogelström, and F. Lichtenberg, Phys. Rev. Lett. 84, 1595 (2000).

[23] Z. Q. Mao, K. D. Nelson, R. Jin, Y. Liu, and Y. Maeno, Phys. Rev. Lett. 87, 037003 (2001).

[24] Y. Tanaka and S. Kashiwaya, Phys. Rev. Lett. 74, 3451 (1995).

[25] S. Kashiwaya and Y. Tanaka, Rep. Prog. Phys. 63, 1641 (2000).

[26] M. Yamashiro, Y. Tanaka, and S. Kashiwaya, Phys. Rev. B 56, 7847 (1997).

[27] S. Kashiwaya, H. Kashiwaya, H. Kambara, T. Furuta, H. Yaguchi, Y. Tanaka, and Y. Maeno, Phys. Rev. Lett. 107, 077003 (2011).
[28] M. Yamashiro, Y. Tanaka, Y. Tanuma, and S. Kashiwaya, J. Phys. Soc. Jpn. 67, 3224 (1998).

[29] C. Honerkamp and M. Sigrist, J. Low Temp. Phys. 111, 895 (1998).

[30] S. Kashiwaya, Y. Tanaka, M. Koyanagi, H. Takashima, and K. Kajimura, Phys. Rev. B 51, 1350 (1995).

[31] S. Kashiwaya, Y. Tanaka, N. Terada, M. Koyanagi, S. Ueno, L. Alff, H. Takashima, Y. Tanuma, and K. Kajimura, J. Phys. Chem. Solid 59, 2034 (1998).

[32] M. Covington, M. Aprili, E. Paraoanu, L. H. Greene, F. Xu, J. Zhu, and C. A. Mirkin, Phys. Rev. Lett. 79, 277 (1997).

[33] L. Alff, H. Takashima, S. Kashiwaya, N. Terada, H. Ihara, Y. Tanaka, M. Koyanagi, and K. Kajimura, Phys. Rev. B 55, R14757 (1997).

[34] J. Y. T. Wei, N.-C. Yeh, D. F. Garrigus, and M. Strasik, Phys. Rev. Lett. 81, 2542 (1998).

[35] I. Iguchi, W. Wang, M. Yamazaki, Y. Tanaka, and S. Kashiwaya, Phys. Rev. B 62, R6131 (2000).

[36] C. R. Hu, Phys. Rev. Lett. 72, 1526 (1994).

[37] M. Sato, Y. Tanaka, K. Yada, and T. Yokoyama, Phys. Rev. B 83, 224511 (2011).

[38] Y. Tanaka and S. Kashiwaya, Phys. Rev. B 70, 012507 (2004).

[39] Y. Tanaka, S. Kashiwaya, and T. Yokoyama, Phys. Rev. B 71, 094513 (2005).

[40] Y. Asano, Y. Tanaka, and S. Kashiwaya, Phys. Rev. Lett. 96, 097007 (2006).

[41] Y. Tanaka, Y. Asano, A. A. Golubov, and S. Kashiwaya, Phys. Rev. B 72, 140503 (2005).

[42] Y. Asano, A. A. Golubov, Y. V. Fominov, and Y. Tanaka, Phys. Rev. Lett. 107, 087001 (2011).

[43] X.-L. Qi and S.-C. Zhang, Rev. Mod. Phys. 83, 1057 (2011).

[44] G. M. Luke, Y. Fudamoto, K. M. Kojima, M. I. Larkin, J. Merrin, B. Nachumi, Y. J. Uemura, Y. Maeno, Z. Q. Mao, Y. Mori, H. Nakamura, and M. Sigrist, Nature (London) 394, 558 (1998).

[45] J. Xia, Y. Maeno, P. T. Beyersdorf, M. M. Fejer, and A. Kapitulnik, Phys. Rev. Lett. 97, 167002 (2006).

[46] M. Matsumoto and M. Sigrist, J. Phys. Soc. Jpn. 68, 3120 (1999).

[47] J. R. Kirtley, C. Kallin, C. W. Hicks, E.-A. Kim, Y. Liu, K. A. Moler, Y. Maeno, and K. D. Nelson, Phys. Rev. B 76, 014526 (2007).

[48] C. W. Hicks, J. R. Kirtley, T. M. Lippman, N. C. Koshnick, M. E. Huber, Y. Maeno, W. M. Yuhasz, M. B. Maple, and K. A. Moler, Phys. Rev. B 81, 214501 (2010).

[49] W. Huang, E. Taylor, and C. Kallin, Phys. Rev. B 90, 224519 (2014). 
[50] S. Lederer, W. Huang, E. Taylor, S. Raghu, and C. Kallin, Phys. Rev. B 90, 134521 (2014).

[51] P. E. C. Ashby and C. Kallin, Phys. Rev. B 79, 224509 (2009).

[52] W. Huang, S. Lederer, E. Taylor, and C. Kallin, Phys. Rev. B 91, 094507 (2015).

[53] A. Bouhon and M. Sigrist, Phys. Rev. B 90, 220511 (2014).

[54] Y. Tada, N. Kawakami, and S. Fujimoto, New J. Phys. 11, 055070 (2009).

[55] S.-I. Suzuki and Y. Asano, Phys. Rev. B 94, 155302 (2016).

[56] T. Hirai, N. Yoshida, Y. Tanaka, J. Inoue, and S. Kashiwaya, J. Phys. Soc. Jpn. 70, 1885 (2001).

[57] T. Hirai, Y. Tanaka, N. Yoshida, Y. Asano, J. Inoue, and S. Kashiwaya, Phys. Rev. B 67, 174501 (2003).

[58] N. Yoshida, Y. Tanaka, J. Inoue, and S. Kashiwaya, J. Phys. Soc. Jpn. 68, 1071 (1999).

[59] H. Li and X. Yang, Solid State Commun. 152, 1655 (2012).

[60] Q. Cheng and B. Jin, Phys. B (Amsterdam, Neth.) 426, 40 (2013).

[61] M. S. Anwar, S. R. Lee, R. Ishiguro, Y. Sugimoto, Y. Tano, S. J. Kang, Y. J. Shin, S. Yonezawa, D. Manske, H. Takayanagi, T. W. Noh, and Y. Maeno, Nat. Commun. 7, 13220 (2016).

[62] P. Gentile, M. Cuoco, A. Romano, C. Noce, D. Manske, and P. M. R. Brydon, Phys. Rev. Lett. 111, 097003 (2013).

[63] D. Terrade, P. Gentile, M. Cuoco, and D. Manske, Phys. Rev. B 88, 054516 (2013).

[64] P. M. R. Brydon and D. Manske, Phys. Rev. Lett. 103, 147001 (2009).

[65] P. M. R. Brydon, Phys. Rev. B 80, 224520 (2009).

[66] S. Wu and K. V. Samokhin, Phys. Rev. B 81, 214506 (2010).

[67] G. E. Blonder, M. Tinkham, and T. M. Klapwijk, Phys. Rev. B 25, 4515 (1982).

[68] See Supplemental Material at http://link.aps.org/supplemental/ 10.1103/PhysRevB.98.014508 for a derivation of the expression of the current through the N/F/S junction.
[69] We note that Eq. (35) is still valid in the presence of spin rotation. SOC influences only the transport coefficients and does not affect the validity of Eq. (35).

[70] Y. J. Chang, C. H. Kim, S.-H. Phark, Y. S. Kim, J. Yu, and T. W. Noh, Phys. Rev. Lett. 103, 057201 (2009).

[71] C.-T. Wu, O. T. Valls, and K. Halterman, Phys. Rev. B 90, 054523 (2014).

[72] The condition under which the Andreev bound states emerge is given by $\Delta\left(k_{z}\right) \Delta\left(-k_{z}\right)<0$ [78]. All pair potentials in Eq. (16), however, do not depend on $k_{z}$. Therefore, no Andreev bound state appears in a junction along the $c$ axis.

[73] There are two types of the zero-energy conductance peaks. One is a conductance peak which appears accidentally at zero energy; the other is a conductance peak which should appear at the zero energy because of, for example, the Andreev interface/surface bound state originating from the topology of the bulk wave functions. A zero-energy peak is not expected on the surface perpendicular to the $c$ direction of $\mathrm{Sr}_{2} \mathrm{RuO}_{4}$. Moreover, if the peak in Fig. 3(a) is caused by, for example, the Andreev bound states in F, the energy of the conductance peak is not affected by the details of a system such as the thickness. In Fig. 3(a), the peaks at $\pm \Delta_{0}$ for $L=0$ move in the $\mp E$ direction with increasing $L$. At $k_{F} L=15$, these two peaks merge. For a longer $L$, the two peaks just pass each other.

[74] K. Yada, A. A. Golubov, Y. Tanaka, and S. Kashiwaya, J. Phys. Soc. Jpn. 83, 074706 (2014).

[75] K. Kawai, K. Yada, Y. Tanaka, Y. Asano, A. A. Golubov, and S. Kashiwaya, Phys. Rev. B 95, 174518 (2017).

[76] M. Fogelström, D. Rainer, and J. A. Sauls, Phys. Rev. Lett. 79, 281 (1997).

[77] Y. Tanaka, Y. Tanuma, K. Kuroki, and S. Kashiwaya, J. Phys. Soc. Jpn. 71, 2102 (2002).

[78] Y. Asano, Y. Tanaka, and S. Kashiwaya, Phys. Rev. B 69, 134501 (2004). 